\title{
Fertilisation and moral status: a scientific perspective
}

\author{
Karen Dawson Monash University, Australia
}

\section{Author's abstract}

The debate about the moral status of the embryo has gained new impetus because of the advances in reproductive technology that have made early human embryo experimentation a possibility, and because of the public concern that this arouses. Several philosophical arguments claiming that fertilisation is the event that accords moral status to the embryo were initially formulated in the context of the abortion debate. Were they formulated with sufficient precision to account for the scientific facts as we now understand them? Or do these arguments need modification? Aspects of three arguments for moral status being acquired at fertilisation are examined in relation to current scientific knowledge, highlighting the reasons why such arguments, at present, seem to provide an inadequate basis for the determination of moral status.

Advances in reproductive technology have made it technically possible for the early human embryo to be an experimental subject. This has enlivened debate concerning the moral status of the prenate (1), for some consensus on this issue is essential for policy formation aimed at regulating the future of such research.

Within the context of the abortion debate, various landmarks in prenatal development are nominated as the determinant of full moral status. Developmentally the earliest of these is fertilisation (2). This paper examines some of the arguments for claiming that fertilisation is the basis for full moral status in the context of current scientific knowledge. Have these arguments been stated with sufficient precision to cope with the facts as we now understand them? Do they need to be modified and if so, how might this be done? But before these questions can be considered some understanding of fertilisation itself is necessary.

\section{What is fertilisation?}

Human fertilisation is a complex process requiring about 24 hours for completion. Viewed simply, it

\section{Key words}

Fertilisation; early human embryo; multiple births; moral status; zygote; individual; genetics; numerical continuity. begins with a spermatozoon, the male gamete, penetrating the ovum or female gamete and culminates in the mingling of the genetic material from each to form a single-celled zygote.

Historically, fertilisation was believed to be possible $\vec{\omega}$ only in the uterine or fallopian tubes of the female, $\mathrm{O}$ but recent medical advances resulting in many births world-wide, have demonstrated that in vitro 0 fertilisation is also possible (3). Regardless of the location of the process, its biological consequences are the same: fertilisation restores the diploid chromosome number, enhances genetic variation, results in sex determination and is a necessary prerequisite for $\vec{\varnothing}$ embryogenesis to proceed (4).

\section{Fertilisation and moral status: the arguments} examined

Arguments in support of fertilisation as the time at $\stackrel{\unrhd}{\perp}$ which full moral status is acquired either rely solely $\overrightarrow{\overrightarrow{0}}$ on features of the fertilisation process, or on some of 3 its aspects in combination with an emphasis on the potential of the newly-formed entity. Those arguments depending on potential have been considered elsewhere (5), so the focus here is only on those arguments that rely on features of the fertilisation process. For the purposes of discussing the relevant biology, these arguments can be considered as three major types: the genetic argument, the discontinuity/ continuity argument and the individuality argument.

\section{The genetic argument}

In essence, this approach pin-points fertilisation as the time at which moral status is acquired as it is then $\underset{\mathrm{C}}{\mathrm{C}}$ that entities that 'are genetically human beings' (6) are created. For this argument the crucial event during

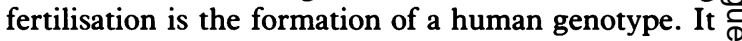
is claimed that only at fertilisation, and not before, $\stackrel{\mathscr{P}}{+}$ does a new genetic member of the species Homo sapiens 7 come about, and at no other point in development is there any 'significant' (7) genetic change. This claim is often coupled with the basic moral principle that it $\mathbb{D}$ is wrong to destroy innocent human beings which, if $\frac{\varrho}{\sigma}$ taken to include the zygote, leads to the conclusion 
that it is wrong to destroy early human life from the moment of fertilisation (8).

Biologically, this view raises two major questions firstly, what constitutes the state of being genetically human and secondly, what is meant by a significant genetic change?

Taking the first question: the genome or genetic make-up of an organism may be considered at three levels - the comparatively gross level of the chromosome, the level of the gene itself and the even finer level of the molecular structure of the gene. If the condition of being genetically human is considered chromosomally, it is either implicit or explicit (9) in this argument that one prerequisite is the presence of 46 chromosomes ( 23 of which are contributed by the egg and 23 by the sperm). Presumably these chromosomes are of an accepted karyotypic configuration for the number of 46 is not unique to humans (10). However, a definition of being genetically human based on chromosome number creates problems - for what then is the status of those who fail to fulfil this requirement?

There are numerous human conditions compatible with postnatal life identifiable by the presence of 45 , 47 or more chromosomes. The most extreme example results from dispermy: two rather than one sperm enter the egg and participate in fertilisation. The result may be the formation of a tripoid zygote (a zygote containing 69 or three sets of chromosomes as opposed to the usual 46 or two sets). Triploidy is estimated to occur in 1-3 per cent of all human fertilisations (11) and in vitro as many as $8-10$ per cent of fertilisations can be observed to result from the penetration of more than one sperm (12). The majority of triploids are spontaneously aborted or still-born but there are some reports of live-born individuals who have lived for up to seven months after birth (13).

More common conditions showing a variation in chromosome number and accompanied by much longer life-spans include Triple-X females (about $1 /$ 1500 live female births), Klinefelter's Syndrome (about 1/500 live male births) and Down's Syndrome (about 1/500 live births) which are usually associated with 47 chromosomes in the karyotype. In contrast to this range of disorders, postnatal existence with only 45 chromosomes is more limited. Turner's Syndrome is the only such chromosomal condition in humans in which one chromosome may be completely absent. The affected females (about 1/4500 live female births) are missing an X-chromosome from the genome (14).

The incidence of these chromosomal conditions at birth is very much lower than at fertilisation, for it is estimated that more than 90 per cent of them are lost through very early pregnancy loss and later spontaneous abortion (15). The possibility of viable individuals, recognised as human, but with 45,47 or even more chromosomes, raises a question about the adequacy of using the criterion of chromosome number rigidly, or at least solely, to define a genetic human being. Some further defining characteristics are needed.

Variation at the level of the gene is inherent in the genetic argument and is extremely common in nature. The simplest example of this 'natural variation' is the occurrence of genetic 'polymorphisms', that is the presence in a population of alternative forms of a gene, known as alleles. This is found, for example, for the major human blood-typing system, the ABO-blood group, where various combinations of the alleles of this gene occur with varying incidences in different populations (16).

A similar degree of variation has also become evident at the even finer level of gene structure since molecular genetics provided an extensive array of techniques that permit fragments of the genetic material, DNA, to be isolated and their chemical composition identified. Use of these techniques, especially restriction enzyme mapping (17), has demonstrated that not all phenotypically equivalent alleles exhibit the same chemical composition in different individuals.

This variation at the genetic level either in the allele present or its chemical composition complicates the definition of an entity as genetically a human being. Where should the boundaries of natural variation be drawn? Can defining a human being solely in genetic terms take account of this range of variation? This type of definition is likely to become increasingly complex as more of the human genome is examined, for at present the genetic content of at most 10 per cent of it is known (18).

Adoption of the claim of Noonan (19) that: '... if you are conceived by human parents you are human' may be seen as a possible means of circumventing the difficulties in defining a genetic human being. In the light of recent advances in in vitro fertilisation (IVF) this approach raises the question: If you are conceived by IVF-procedures, from human material, do you still qualify as human? An affirmative answer here surely suggests that the origin of the material rather than any of its characteristics is what is important for specifying a human being. However, even this possible solution may be short-lived, for it relies on species breeding true to their kind. But consider the situation of transgenic animals.

A transgenic animal is one carrying a gene from another species, such as a mouse with a gene from a human source (20). How many human genes can be introduced into a phenotypic mouse before it is considered as genetically non-mouse or even human? Is it the intra-uterine existence of the entity within a mouse that ensures that such a transgenic animal remains a mouse? Alternatively, would the introduction of a gene from an animal into a human gamete or early embryo, say for the purposes of gene therapy, invalidate the humanity of any offspring produced subsequently? What if many animal genes were to be introduced? The advent of transgenic animals blurs the boundaries of the intended meaning of species as 'a discrete breeding unit' and emphasises the need for criteria other than those of the genetic 
content and its origin, and the site of fertilisation being applied in attributing human, and hence moral, status to the prenate. The question 'What is a genetic human being?' does not have a simple answer. From available scientific data it is presently essentially indefinable.

The second part of the genetic argument emphasises fertilisation as the time of acquiring moral status because no further 'significant' genetic changes occur beyond this time. The meaning of 'significant' is not clearly specified in the literature but genetic changes can and do occur after fertilisation - are they significant?

Extreme genetic changes occur during the differentiation of erythrocytes (red blood cells) and cells of the lens of the eye. When finally differentiated these cells contain no nuclear genetic material at all, and conversely, there is a low incidence of cells in the liver that double their genetic content during differentiation (21). Thus, the postnatal human may be considered as composed of various families of cells some of which have been irreversibly genetically changed during development subsequent to fertilisation.

Further genetic changes may also occur throughout the pre- and postnatal phases as a result of randomly occurring heritable changes in the genetic material known as spontaneous mutation. The significance of these random changes depends on their location in the DNA (22), the stage of development at which they occur (23) and their nature (24). Mutation is estimated to occur spontaneously at a rate of one per million base pairs per round of DNA replication although this rate differs for specific genes.

It may be argued that the above genetic changes are simply 'variations on a theme' for they are qualitative changes following fertilisation, but there are also changes that can occur at this time that alter the genetic make-up of the prenate. These changes result from chromosomal non-disjunction, that is failure of the chromosomes to separate properly during cell division. The result of non-disjunction during the first division of the zygote is a mosaic organism, that is an organism composed of two different cell lines, both of which differ genetically from the zygote from which they arose. If non-disjunction occurs at a later stage of development it will theoretically result in the formation of an individual with three different cell lines, two of which differ genetically from the zygote. The chromosomal conditions mentioned previously (Triple-X, Klinefelter's Syndrome, Down's Syndrome and Turner's Syndrome) may exist in either a mosaic form, resulting from chromosomal non-disjunction following fertilisation, or a pure form from a nondisjunction event during gametogenesis in either parent prior to fertilisation. The genetic changes here involve the gain or loss of one whole chromosome after fertilisation which may have gross effects on the phenotype, genotype and survival of the developing prenate and should consequently be considered as significant.
In summary, the questions arising from the genetic $\stackrel{\frac{1}{2}}{2}$ argument stem mostly from viewing genetic make-upm as something static and constant from the moment of $\overrightarrow{\bar{\nabla}}$. fertilisation. In reality, differentiation is a dynamic? process and the environment contributes to subtle $\vec{F}$ continuous changes throughout a life-time. Some acknowledgement of this needs to be made in arguments emphasising genotype formation as an $\frac{\bar{c}}{\bar{c}}$ important aspect of fertilisation determining the moral $\frac{\widetilde{\sigma}}{\sigma}$ status of the prenate.

\section{The discontinuity-continuity argument}

Proponents of this argument (25) view events post fertilisation as comprising a continuum of $\vec{\omega}$ developmental changes, such that it is impossible to isolate any one stage at which to attribute the attainment of moral status. In contrast to this $\frac{\dot{\omega}}{\omega}$ continuity, fertilisation is seen as a radical ${ }_{\infty}^{\omega}$ discontinuity or 'transformation' (26) in development. It is then argued that the union of the two gametes tow form the single zygote at fertilisation is the only음 discrete stage at which it can be claimed that a human entity begins to exist.

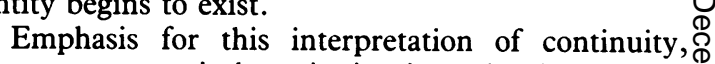
known as numerical continuity, is on the change from 3 two gametes to one zygote that is continuous $\stackrel{\Phi}{\square}$ throughout all following development (27). As previously mentioned, in the formation of triplogs $\infty$ individuals more than two entities may sometimes. participate in fertilisation. A further deviation of the occurs with parthenogenesis - development of the eg్g without fertilisation by a sperm. At present this holds only little relevance to human reproduction, as so far as is known no births have resulted from this process, $\stackrel{\varnothing}{\unrhd}$ although the initial stages of parthenogenetic $\vec{F}$ development have been observed rarely in vitro (28). 3 Here, there is no numerical discontinuity unless, as suggested by Quinn (26) the environmental agent? inducing parthenogenetic development is treated as a

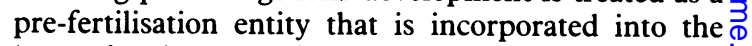
'zygote' at the onset of development. But this approach $\frac{\sigma}{3}$ implies that environment is irrelevant in the events of normal fertilisation, which leads to an arbitrary and $ᄋ$ unsatisfactory distinction not reflecting the actual course of events.

Dispermy and parthenogenesis represent different $\rightarrow$ deviations in the number of entities participating in the process leading to the initiation of development $\bar{N}$ which may be able to be incorporated into the notion o of numerical continuity. But even if these fluctuations $N$ are accepted, the notion also specifies that the result $\underset{\omega}{N}$ of fertilisation is the formation of a single entity - the zygote. Is this necessarily the case?

Consider the outcome of dispermy. If dispermy occurs it may predispose to a tumorous condition $\stackrel{\infty}{+}$ known as a hydatidiform mole (29). In such an event $\square$ embryonic development may not occur. The medical $\stackrel{\vec{D}}{\mathrm{D}}$ concern with the formation of moles is that they may $\stackrel{\odot}{\stackrel{\Phi}{\perp}}$ become malignant and life-threatening for the mother, $\stackrel{\mathbb{Q}}{\complement}$ but here they serve as an example of how fertilisation 
may not necessarily lead to the formation of a zygote.

Now, consider again the outcome of fertilisation. The one zygote present in the context of this argument, is said to mark the beginning of a human entity that is numerically continuous throughout all subsequent development. Is this the case? What if the zygote should split soon after its formation? Can the notion of numerical continuity cope with the possibility?

Identical twins arise from a single zygote that splits. The mechanism involved is not important here, the point is that with this type of twinning there is only temporary numerical discontinuity, ie 1 egg +1 sperm $\rightarrow 1$ zygote $\rightarrow 2$ individuals. In trying to make numerical continuity allow for twinning Quinn (26) concluded that identical twinning, if environmentally determined, was a developmental abnormality. Such an approach is analogous to that used when attempting to reconcile parthenogenesis with numerical continuity for the net result is to discount any possible role environment may play in singleton zygote development. At present, the relative contribution of genotype and environment to identical twinning is unclear. Identical twins occur in about one of every 270 pregnancies coming to term (4), and it has been observed that a proportion of identical twins are lost either through spontaneous abortion (30) or the loss of one fetus which results in singleton development and birth (31). Further studies of twins during gestation may show identical twins to be more frequent than currently believed. The techniques for such studies to proceed are now becoming available and if a genetic component to identical twin formation could be demonstrated their status, as seen by Quinn, as a 'developmental abnormality' would need reappraisal.

The concept of numerical continuity is too narrow as initially defined to allow for any variations during or subsequent to fertilisation. To be ranked as a valid determinant of moral status some refinement of the concept, incorporating current scientific knowledge is needed.

\section{The individuality argument}

Proponents of this argument also claim that fundamental moral principles against killing are applicable from fertilisation, as this event marks the time when an individual human being begins to exist. The meaning of individual differs among authors. An emphasis on genetics is sometimes integral to the notion:

$\because .$. the unique genetic package of an individual is laid down at fertilisation' (32)

while others highlight continuity:

'. . it is the same individual right through from that moment (fertilisation) on to the end' (33).

The problems with these views have largely been discussed. The genotype of an individual later in life is not necessarily that formed at fertilisation; many changes can occur subsequently. Similarly, the individual created at fertilisation may not remain the same throughout life. The simplest demonstration of this is identical twinning which is possible for about 12 days after fertilisation. In this process the original zygote ceases to exist. Conversely, during this time it is also possible for two zygotes derived from the independent fertilisation of two eggs to fuse forming a chimera - the one individual resulting from two fertilisation events. In neither of these cases is the developing individual the one that was formed at fertilisation.

Other authors appeal to the potential of the zygote:

'We know that a new human individual organism with the internal potential to develop into an adult ... comes into existence as a result of the process of fertilisation ...'(34).

The intention is not to discuss potential here. However, consideration of this argument does illustrate one feature inherent in many arguments in support of fertilisation: a reliance on the viability of the zygote. It is worthwhile noting that for up to 78 per cent of human fertilisations the end-point is loss (35) rather than progression to the next developmental stage which can be seen as weakening the applicability of any argument depending on viability.

\section{Summary}

Scientific facts alone cannot provide the answer to the debate on the moral status of the prenate. The final outcome is an ethical decision. However, information about the relevant biological events can create a firmer basis for discussion of such a clearly interdisciplinary issue. Assessment of the arguments claiming that fertilisation is the time at which full moral status is acquired is now extremely relevant as policy-formation and legislation for the regulation of reproductive technology and pre-embryo research is considered in many countries.

The arguments discussed basically rely on the simple equation that: $1 \mathrm{egg}+1$ sperm $=1$ zygote $=$ 1 child.

This process is seen as marking the beginning of a human life, and thus determining that fertilisation is the time at which moral status is accorded. The point of whether or not fertilisation is the beginning of human life will not be debated here. Leaving this aside, it seems that overall the different arguments for fertilisation determining moral status of the prenate oversimplify actual biological events.

Among the problems encountered are an overemphasis on the role of genetics in directing the course of events after fertilisation, and a dependence on the fidelity of the new genotype formed at fertilisation throughout all subsequent development. Also sometimes inherent in this argument is the 
assumption that birth will follow from fertilisation. Several instances where biology diverges from these claims have been discussed and many more equivalent examples could be cited.

When assessing the claim that fertilisation establishes full moral status, several'facts should be kept in mind:

1) Given suitable environmental conditions, development may sometimes commence without fertilisation occurring (parthenogenesis).

2) The genotype of any individual may not be that formed at fertilisation.

3) Development and differentiation after fertilisation result in changes to the genetic complement of the prenate.

4) Environment is a potent force in the course of development both prenatally and postnatally.

5) The formation of a single zygote at fertilisation may be the forerunner of the development of multiple individuals that may or may not be genetically identical.

6) Successful completion of fertilisation in no way assures development through to birth or even the commencement of embryo development.

Until arguments claiming fertilisation as the determinant of moral status take into account such facts by being modified to incorporate them, they provide an inadequate basis for policy-formation or legislation regulating reproductive technology as they hold only little relevance to actual biology. Presently, this situation serves to raise the questions of whether the whole issue of moral status needs reappraisal and whether any legislation, either actual or proposed, in this area is premature.

\section{Acknowledgements}

This work was supported by an NH and MRC Special Initiative Grant to Professor J Swan, Professor L Waller, Dr M Brumby and Dr H Kuhse who provided helpful discussion of this manuscript. Special thanks to Ms L Fleming who provided some of the philosophical material and Professor P Singer for his helpful criticism and comments.

Karen Dawson gained her Phd in Genetics, from La Trobe University, Victoria, in 1981. Following this she spent four years as a Senior Tutor in the Genetics Department, Monash University, teaching Genetics to science and medical undergraduates. In 1985 she took up her current position as Scientific Research Officer in the Centre for Human Bioethics and is currently involved in a research project concerned with ethical issues arising from reproductive technology.

\section{References and notes}

(1) 'Prenate', in this paper, is used as a term for all prenatal stages of development thus reserving the specific terms 'pre-embryo', 'embryo' and 'fetus' for proper usage when applicable.

(2) The term 'fertilisation' is used throughout in preference to 'conception'. As has been noted 'conception' has become an ambiguous term that is increasingly associated with implantation, rather than the specific union of the sperm and ovum that is denoted by fertilisation. For example see Federal Republic of Germany, Judgement at Karlsruhe. In: Ethical aspects of abortion: some European views. IPPF Report. Appendix 2. 1975.

(3) The number of births from IVF is increasing daily. The highest estimate thus far is in excess of 2000, according to Dr Howard Jones at the 4th World Conference on In vitro fertilisation held in Melbourne, Australia in November, 1985.

(4) Moore K. The developing human (3rd ed). Philadelphia: W B Saunders and Co, 1982.

(5) Singer P, Dawson K. IVF technology and the argument from potential (in preparation).

(6) Werner R. Abortion: the moral status of the unborn. Social theory and practice, 1974; 3:201-222.

(7) See reference (6): 202.

(8) R Werner is the chief proponent of this argument but similar arguments are also provided, by for example, $\mathrm{R}$ Wertheimer: Understanding the abortion argument. Philosophy and Public Affairs 1971; 1: 67-95; Brody B. On the humanity of the fetus. In: Beauchamp T R, Walters L, eds. Contemporary issues in bioethics California: Wadsworth, 1978: 229-240, and Santamaria $P$ In vitro fertilisation and embryo transfer. In: Brumby $M$ N ed. Proceedings of the conference: In vitro fertilisation problems and possibilities. Clayton, Victoria: Centre for Human Bioethics, Monash University, 1982: 48-53.

(9) For example see Noonan J T. An almost absolute value in history. In: Noonan J T, ed. The morality of abortion. Harvard: Harvard University Press, 1970: 1-59.

(10) Hsu TC and Benirschke K. An atlas of mammalian chromosomes (Vol 10). New York: Springer-Verlag, 1977.

(11) Jacobs P A, Angell R R, Buchanan I M, Hassold T J, Matsuyama A M and Mannel B. The origin of human triploids. Annals of human genetics 1975; 42: 49-57.

(12) Plachot M, Mantelbaum J, Junca A, Salns-Baroux J and Cohen J. Impairment of human embryo $\frac{5}{3}$ development after abnormal in vitro fertilisation. In: Seppälä M, Edwards R G, eds. In vitro fertilisation and $\delta$ embryo transfer. Annals of the New York Academy of Sciences 1985; 442: 336-341.

(13) Schinzel A. Catalogue of unbalanced chromosome aberrations in man. New York: Walter de Gruyter, 1984.

(14) Wilson M G. Cytogenetics update for pediatricians. Current problems in pediatrics $1985 ; 15: 1-47$.

(15) Boué A, Boué J, Bropp A. Cytogenetics of pregnancy wastage. Advances in human genetics 1985; 14: 1-59.

(16) See Bodmer W F, Cavalli-Sforza L L. Genetics, evolution N and man. San Francisco: W H Freeman, 1976, for a full $O$ discussion of racial and population differences.

(17) For a general discussion of restriction mapping see Norvick R P. Plasmids. Scientific American 1980; $\underset{\oplus}{\stackrel{\oplus}{\hookrightarrow}}$ 243:76-91.

(18) McKusick V A. The human gene map. Clinical genetics 1984; 27:207-239.

(19) See reference (9): 51.

(20) Palminter R D, Brinster R L. Transgenic mice. Cell $\stackrel{\mathbb{Q}}{\stackrel{8}{\circ}}$

(21) Davidson E H. Gene activity in early development. New 
York: Academic Press, 1977.

(22) Much of the DNA in humans is apparently never used for the production of proteins (the usual gene product) and so spontaneous mutations in this genetic material will have no effect on the phenotype of the organism. Also, the genetic material that is read for protein production is read as a triplet code and the code is such that any mutation in the third base of a triplet will not always affect the protein being produced. In contrast a mutation in the first or second positions of a triplet may have a range of effects on the protein being produced, and may even stop its production.

(23) Some genes are only functional at certain stages of development and once that stage is past remain switched off. One instance of this is in the production of human haemoglobin where different clusters of genes are active during the embryo phase, fetal phase, and child and adult phase. Mutations during adult life in the embryo phase gene complex would have no effect on the adult, as these genes are then inactive.

(24) Mutations are of various types - deletion, insertion, base substitution, inversion and duplication - which may involve only small regions of the genetic material. There are also relatively large-scale structural changes such as transposition, inversion, translocation and duplication which have major effects on the function of the genome.
(25) See for example Grisez G C. Abortion: the myths, the realities and the arguments. New York: Corpus Books, 1970. Iglesias T. In vitro fertilisation: the major issues. Fournal of medical ethics 1984: 1: 32-37 and also reference (8).

(26) Quinn W. Abortion: identity and loss. Philosophy and public affairs 1984; 13: 24-54.

(27) See references (6) and (26)

(28) Edwards R G, Trounson A. Discussion on the growth of human embryos in vitro. In: Edwards R G, Purdy J $M$, eds. Human conception in vitro. London: Academic Press 1982: 219-233.

(29) Snell R S. Clinical embryology for medical students (2nd ed). London: Little Brown and Co, 1972.

(30) Morison J E. Foetal and neonatal pathology (2nd ed). London: Butterworths, 1968.

(31) Walters W A W, Renou P M. Pregnancy care. In: Wood C, Trounson A, eds. Clinical in vitro fertilisation. New York: Springer-Verlag, 1984: 147-156.

(32) See reference (8) Santamaria J: 49.

(33) Daly T. Discussion in Conference Proceedings. In vitro fertilisation: problems and possibilities. Clayton, Victoria: Centre for Human Bioethics, Monash University. 1982: 62.

(34) See reference (25) Iglesias T: 36.

(35) Roberts C J, Lowe C R. Where have all the conceptions gone? Lancet 1975; 1:498-499.

\section{News and notes}

Institute of Medical Ethics

London Medical Group

\section{5th annual conference Children at risk}

The conference will examine moral dilemmas in the treatment and abuse of children by parents and doctors.

Date and place:

Friday and Saturday 12 and 13 February 1988 at the Royal College of Surgeons of England, Lincoln's Inn Fields.

Conference fee: $£ 75$ (IME members $£ 60$, students $£ 10$ ).

Details and application form (SAE PLEASE) from the Conference Secretary, London Medical Group, PO Box 20, Tavistock House North, Tavistock Square, London WC1H 9LG (tel 01-387 8132). 